\title{
PARAMETRIC INTEGRAL EQUATIONS SYSTEMS METHOD IN SOLVING UNSTEADY HEAT TRANSFER PROBLEMS FOR LASER HEATED MATERIALS
}

\author{
Dominik SAWICKI*, Eugeniusz ZIENIUK**
}

*Faculty of Mechanical Engineering, Bialystok University of Technology, ul. Wiejska 45C, 15-351 Białystok, Poland

${ }^{* *}$ Faculty of Mathematics and Computer Science, University of Bialystok, ul. Konstantego Ciołkowskiego 1M, 15-245 Białystok

sawicki.dominik1@gmail.com, ezieniuk@ii.uwb.edu.pl

received 11 April 2015, revised 26 October 2015, accepted 28 October 2015

\begin{abstract}
One of the most popular applications of high power lasers is heating of the surface layer of a material, in order to change its properties. Numerical methods allow an easy and fast way to simulate the heating process inside of the material. The most popular numerical methods FEM and BEM, used to simulate this kind of processes have one fundamental defect, which is the necessity of discretization of the boundary or the domain. An alternative to avoid the mentioned problem are parametric integral equations systems (PIES), which do not require classical discretization of the boundary and the domain while being numerically solved. PIES method was previously used with success to solve steady-state problems, as well as transient heat transfer problems. The purpose of this paper is to test the efficacy of the PIES method with time discretization in solving problem of laser heating of a material, with different pulse shape approximation functions.
\end{abstract}

Key words: PIES Method, Transient Heat Transfer, Laser Heating, FEM

\section{INTRODUCTION}

High power lasers found broad application in processing and treatment of materials. They are very popular because of their unique properties, like high degree of special coherence, temporal dependence and beam directivity (Jewtuszenko et al., 2009; Gladush and Smurov, 2011). One of the laser applications is heating up the material in order to change its properties. This process depends highly on the properties of the processed material and the laser itself, therefore modeling and simulation of this phenomenon plays a key role in proper selection of these properties. Analytical methods can be used to simulate these problems (Brugger, 1972; Warren and Spark, 1990; Al-Nimr et al., 2002; Yanez et al., 2002), but they are rather used to solve problems defined with elementary shape areas and elementary boundary conditions. In more complex cases numerical methods are used. The most popular methods are finite element method (FEM) (Lewis et al., 1996) and boundary element method (BEM) (Brebbia te al., 1984; Tanaka et al., 1994; Majchrzak, 2001). FEM requires discretization of the whole domain, while BEM requires only discretization of the boundary. However, when using classical BEM for solving temperature equation, domain integral appears. Numerical integration over the domain requires dividing it into subdomains called cells. Dividing domain into cells in BEM is basically the same as dividing it into elements in FEM, so it loses its main advantage. There are some approaches that allow avoiding domain integrals, like dual reciprocity boundary element method (DRBEM) (Partridge et al., 1992) and multiple reciprocity boundary element method (MRBEM) (Nowak and Brebbia, 1989). However, these modifications have some limitations and cannot be used in all situations. Beside the large number of methods that already exist, it is still very important to search for new ones that would eliminate the disadvantages of the existing methods. An alternative approach is served by a group of methods called meshless methods (Johanssona and Lesnicb, 2008; Xiaokun et al., 2011; Jirousek et al., 1996). These methods require only a number of nodes located inside the domain and on the boundary. Over the past years a method based on the parametric integral equations systems (PIES) has been developed. PIESs are analytical modifications of the classical boundary integral equation (BIE) that allows solving problems without the need of discretization of boundary and domain. It uses Bézier or B-spline curves and patches for modeling. For time dependent problems it also allows to avoid the time discretization, but further research is needed. First, a version of PIES using time discretization should be properly tested. Authors found it interesting to test the method for time dependent boundary conditions.

This paper presents PIES method with time stepping scheme for laser heating of homogeneous materials. Results obtained with PIES have been compared with exact solution and results obtained with FEM.

\section{PIES METHOD FOR UNSTEADY HEAT TRANSFER PROBLEMS}

The differential equation for unsteady Fourier heat conduction, without internal heat sources, is governed by Brebbia et al. (1984) and Majchrzak (2001):

$c \frac{\partial T(\mathrm{x}, t)}{\partial t}=K\left(\frac{\partial^{2} T(\mathrm{x}, t)}{\partial x_{1}^{2}}+\frac{\partial^{2} T(\mathrm{x}, t)}{\partial x_{2}^{2}}\right)$,

where: $K[W / m K]$ is the thermal conductivity, $T(\mathbf{x}, t)$ is the timedependent temperature field, $c\left[J / m^{3} K\right]$ is the volume-specific 
heat capacity, $t$ is time.

The equation (1) is complemented by the given boundary conditions

$\left\{\begin{array}{l}x \in \Gamma_{1}: T(x, t)=T_{s}, \\ x \in \Gamma_{2}: q(x, t)=-K \frac{\partial T}{\partial n}=q_{s},\end{array}\right.$

where: is the given temperature on the boundary segment $\Gamma_{1}, q_{s}$ is the given heat flux on the boundary segment $\Gamma_{2}$ and $n$ is the normal vector.

An initial condition is given by

$\mathrm{x} \in \Omega: \mathrm{T}(\mathrm{x}, 0)=T_{0}(\mathrm{x})$,

where $T_{0}$ is the given temperature inside the domain $\Omega$ at time $t=0$.

Classical boundary integral equation (BIE) for (1) is presented in the form of Majchrzak (2001):

$\alpha(\mathrm{x}) T\left(\xi, t^{F}\right)=-\frac{1}{c} \int_{t^{0}}^{t^{F}} \int_{\Gamma} T^{*}\left(\xi, \mathrm{x}, t^{F}, t\right) q(x, t) d \Gamma d t$

$+\frac{1}{c} \int_{t^{0}}^{t^{F}} \int_{\Gamma} Q^{*}\left(\xi, \mathrm{x}, t^{F}, t\right) T(x, t) d \Gamma d t$

$+\iint_{\Omega} T^{*}\left(\xi, \mathrm{x}, t^{F}, t^{0}\right) T\left(\mathrm{x}, t^{0}\right) d \Omega(\mathrm{x})$,

where $t^{0}, t^{F}$ is the analyzed time interval and

$\alpha(\xi)=\left\{\begin{array}{l}1 \quad \xi \in \Omega \\ 0.5 \text { for } \xi \in \Gamma, \text { for a smooth boundary. } \\ 0 \quad \xi \in \Omega\end{array}\right.$

Integrands $T^{*}$ and $Q^{*}$ from equation (2) can be found in an explicit form in Majchrzak (2001).

After applying analytical modification to the classical BIE, similar to the one applied for steady problems [16], parametric integral equation system (PIES) (3) for unsteady heat transfer problem has been obtained (Zieniuk et al., 2014).

$0.5 T_{l}\left(s_{1}, t^{F}\right)=$

$=\frac{1}{c} \int_{t^{0}}^{t^{F}} \sum_{j=1}^{n} \int_{\bar{s}_{j-1}}^{\bar{s}_{j}} \bar{T}_{l j}^{*}\left(s_{1}, s, t^{F}, t\right) q_{j}(s, t) J_{j}(s) d s d t$

$-\frac{1}{c} \int_{t^{0}}^{t^{F}} \sum_{j=1}^{n} \int_{\bar{s}_{j-1}}^{\bar{s}_{j}} \bar{Q}_{l j}^{*}\left(s_{1}, s, t^{F}, t\right) T_{j}(s, t) J_{j}(s) d s d t$

$+\iint_{\Omega} \overline{\bar{T}}_{l j}^{*}\left(s_{1}, y, t^{F}, t^{0}\right) T\left(y, t^{0}\right) d \Omega(y)$

where: $\quad \bar{s}_{l-1} \leq s_{1} \leq \bar{s}_{l}, \quad \bar{s}_{j-1} \leq s \leq \bar{s}_{j}, \quad l=1,2,3, \ldots, n$. $\bar{s}_{l-1}, \bar{s}_{l} \bar{s}_{j-1}, \bar{s}_{j}$ is the beginning and the end of segments, respectively $S_{l}$ and $S_{j}$, and $J_{j}(s)$ is the Jacobian of the transformation.

Integrands $\bar{T}_{l j}{ }^{*}, \bar{Q}_{l j}{ }^{*}$ and Integral Identity $\bar{T}$ used for obtaining results inside domain have been presented in an explicit form in Zieniuk et al. (2014).

\subsection{Numerical solution of PIES}

To solve PIES for transient heat transfer problem (3), a strategy known from BEM and tested previously in PIES (Zieniuk et al.,
2014) was used. This strategy involves discretization of the time variable and use of the time stepping scheme, with time step size $\Delta t$ in order to get results at desired time. Collocation method was used to obtain an algebraic equations system, according to the algorithm provided in Zieniuk et al. (2014). Number of collocation points should be equal to the number of unknown coefficients in the series, used to approximate unknown boundary functions. After solving the algebraic equations system, unknown coefficients from approximating series are obtained. Presented approaches have been used before, to solve potential problems (Zieniuk, 2013) and lately also problems of transient heat transfer (Zieniuk et al., 2014).

\section{LASER BEAM CHARACTERISTICS}

The main function of the laser in material processing is to generate heat in the processed piece of material. A laser beam can be characterized with set of parameters, like divergence, radius, temporal structure and spatial intensity profile. The efficiency with which a material absorbs an incoming laser beam and converts it to heat depends on the properties of the material, like absorption coefficient, but also on the characteristics of laser beam itself (Jewtuszenko et al., 2009). In the general form, the total laser intensity can be written as

$\mathrm{q}(\mathrm{r}, \mathrm{t})=A q_{0} \varphi(r) q_{t}(t)$,

where $A$ is the effective absorption coefficient of the heated material, $\varphi(r)$ describes the (arbitrary) intensity distribution (beam shape) in the cross-section, $q_{t}(t)$ is the temporal dependence (pulse shape) and $q_{0}$ is the characteristic intensity of the laser (Gladush and Smurov, 2011).

Assuming the spatial intensity profile of the beam to be evenly distributed, equation (4) can be presented as

$\mathrm{q}(\mathrm{t})=A q_{0} q_{t}(t)$.

Laser pulse shape $q_{t}(t)$ is often approximated by simple functions, like (Jewtuszenko et al., 2009):

- Rectangular pulse shape:

$q_{t}(t)=\left\{\begin{array}{lll}1 & \text { for } & 0<t \leq t_{s} \\ 0 & \text { for } & t_{s}<t\end{array}\right.$

- Triangular pulse shape:

$q_{t}(t)=\left\{\begin{array}{lll}2 \frac{t}{t_{r}} & \text { for } & 0<t \leq t_{r} \\ 2 \frac{\left(t_{s}-t\right)}{\left(t_{s}-t_{r}\right)} & \text { for } & t_{r}<t \leq t_{s} \\ 0 & \text { for } & t_{s}<t\end{array}\right.$

where $t_{r}$ is the pulse rise time and $t_{s}$ is the complete pulse time.

For more accurate approximation, Gaussian function can be used (Jewtuszenko et al., 2009):

$q_{t}(t)=\operatorname{Iexp}\left[-\gamma\left(t^{\delta}-t_{r}^{\delta}\right)\right]\left(\frac{t}{t_{r}}\right)^{\beta}, t>0$.

where parameters $\beta, \gamma$ and $\delta$ are related to the pulse rise time $t_{r}$, while value of parameter $I$ can be obtained from the condition of total energy conservation for distributions (6-8).

Fig. 1 presents $q_{t}(t)$ function diagram with $t_{r}=0.2671[s]$ and $t_{s}=1[s]$, for three different laser pulse shape approximation functions (6-8). 


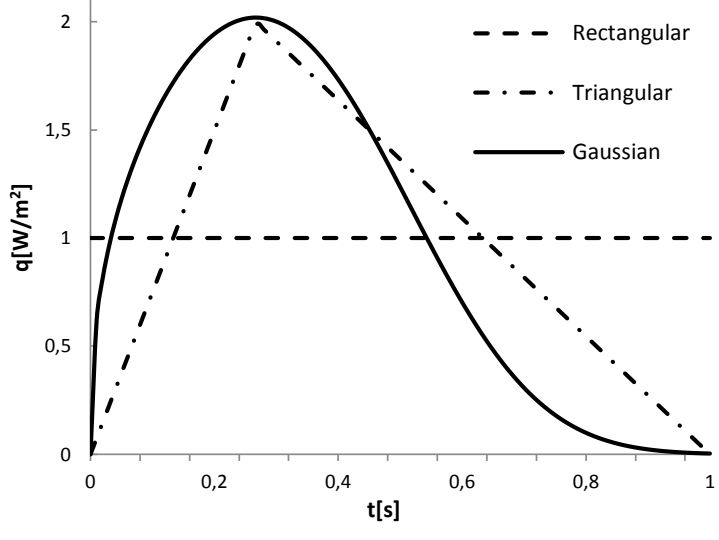

Fig. 1. Diagram of function $q_{t}(t)$ with $t_{r}=0.2671[s]$ and $t_{s}=1[s]$, for three different laser pulse shape approximation functions (7-9)

This paper presents heating of a Steel 45 material with Nd:YAG (neodymium-doped yttrium aluminum garnet) laser, for three different laser pulse shape approximation functions (6-8).

\section{NUMERICAL ANALYSIS}

Few examples have been solved to test efficiency of PIES method for issues simulating laser heating of a material, where all boundary conditions are dependent on time. Results obtained with PIES method have been compared to exact solution and results obtained with FEM. For FEM solution authors have used ANSYS Workbench 14 simulation tool called Transient Thermal. In FEM, square elements with quadratic shape function have been used to model the issue.

Accuracy and stability of results in PIES is dependent on many variables, like time step size $\Delta t$, number of collocation points used in the approximating series and their location, and number of weight coefficients in quadrature for integration over boundary and domain. There is a certain relationship between the time step size $\Delta t$ and the number of weights in the quadrature over the domain in PIES (Zieniuk et al., 2014). The shorter the time step the larger number of weight coefficients has to be taken for integration over the domain. The applied FEM software uses the implicit time integration method, so it is unconditionally stable and does not apply Courant-Friedrich-Levy condition (Lewis et al., 1996). Taking into account the relationship in PIES and unconditionally stable FEM, both methods have been compared for the same time step sizes and a similar number of weight coefficients in quadrature for integration over the domain in PIES and nodes in FEM.

\subsection{Rectangular pulse shape}

First, a symulation of rectangular pulse shape approximation function (6) has been conducted. Exact solution of this problem is presented in the following form (Jewtuszenko et al., 2009):

$\mathrm{T}(\mathrm{y}, \mathrm{t})=A q_{0} \frac{2 \sqrt{k t}}{K} \operatorname{ierfc}\left(\frac{y}{2 \sqrt{k t}}\right), y \geq 0, t \geq 0$,

where $k=K / c$ is the diffusion coefficient and $\operatorname{ierfc}(x)$ is the integral of the complementary error function.

In the exact solution a laser heating of the half-space is considered. However, the half-space has to be approximated with a rectangular area for numerical calculations with PIES and FEM, as shown in Fig. 2.

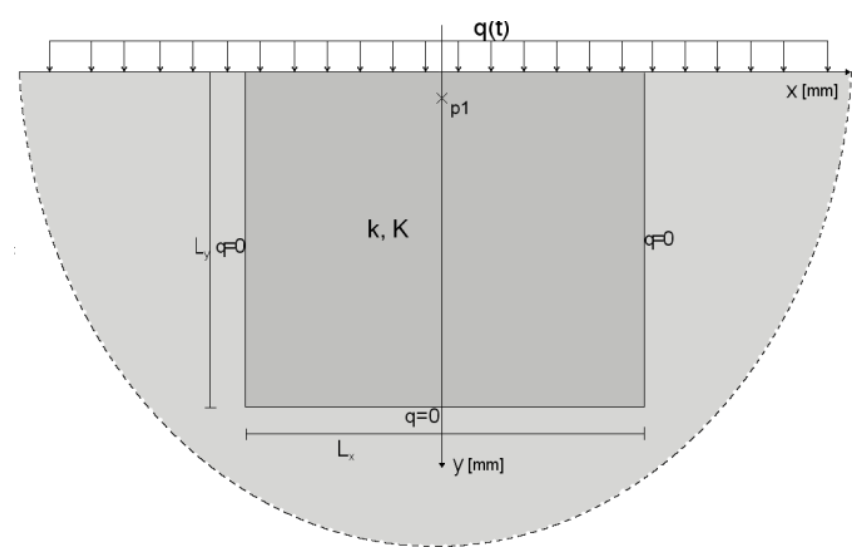

Fig. 2. Laser heating schema

Value of $q(t)$ has been calculated from equation (5). The energy losses by radiation and convection on the surface have been omitted. Material and laser parameters used in calculations are presented in Tab. 1.

Tab. 1. Parameters used for simulation of temperature distribution in material heated by laser beam (Jewtuszenko et al., 2009)

\begin{tabular}{|c|c|c|c|c|l|c|l|}
\hline Metal & Laser & $\begin{array}{l}\mathbf{t}_{\mathbf{s}} \\
{[\boldsymbol{m} \boldsymbol{s}]}\end{array}$ & $\begin{array}{l}\mathbf{t}_{\mathbf{r}} \\
{[\boldsymbol{m} \boldsymbol{s}]}\end{array}$ & $\begin{array}{c}\mathbf{q}_{\mathbf{0}} \times \mathbf{1 0}^{-\mathbf{9}} \\
{\left[\boldsymbol{W m}^{-\mathbf{2}}\right]}\end{array}$ & $\begin{array}{l}\mathbf{K} \\
{\left[\boldsymbol{W m}^{-\mathbf{1}} \boldsymbol{K}^{-\mathbf{1}}\right]}\end{array}$ & $\begin{array}{l}\mathbf{k} \times \mathbf{1 0}^{\mathbf{5}} \\
{\left[\boldsymbol{m}^{\mathbf{2}} \boldsymbol{s}^{-\mathbf{1}}\right]}\end{array}$ & $\begin{array}{l}\boldsymbol{A} \\
{[\%]}\end{array}$ \\
\hline $\begin{array}{c}\text { Steel } \\
45\end{array}$ & Nd:YAG & 1 & 0.2671 & 0.58 & 33.5 & 1.5 & 41 \\
\hline
\end{tabular}

The first step was to establish the optimal size of the area, $L_{x}$ and $L_{y}$ approximating the half space (Fig. 2). Height of the area $L_{y}$ has been determined based on the exact solution (9). A minimal height y has been assumed, for which the temperature $T$ and heat flux $q(t)$ are approximately equal to 0 , for the given boundary condition (5) and maximum simulation time $(t=2 \mathrm{~ms})$. Width of the area $L_{x}$ was established after a series of numerical experiments with use of PIES method. A minimal width $L_{x}$ was sought, for which a further increase of this width won't have any impact on the results obtained at point $p 1(0,0.04)$. It has turned out that the optimal size of approximating area is $L_{x}=L_{y}=3$. It has been also established, that in order to get stable and accurate results there has to be a minimum of 9 collocation points evenly distributed on each of the boundary segments. Also a minimum number of 20 weight coefficients in quadrature for integration over each boundary segment has to considered.

In the next step, the example has been solved multiple times using both methods, PIES and FEM. Results have been obtained for different time step sizes, and different number weight coefficients in quadrature for integration over the domain in PIES and number of nodes in FEM. Results have been compared with the exact solution. Temperature has been measured at point $p 1(0,0.04)$. Tab. 2 presents parameters of different PIES solutions. Results are shown in Fig. 3.

As it can be seen in Fig. 3, PIES method gives more accurate results when decreasing the time step size and increasing the number of weight coefficients in quadrature for integration over the domain. The best results (PIES-3) have been obtained for the smallest time step size and the largest number of weights. 
Next, results obtained with FEM, have been compared with the exact solution. Input data for FEM are shown in Tab. 3. Results are presented in Fig. 4.

Tab. 2. Input data for PIES

\begin{tabular}{|l|c|c|c|}
\hline & PIES-1 & PIES-2 & PIES-3 \\
\hline $\begin{array}{l}\text { weights in the } \\
\text { domain integral }\end{array}$ & 10080 & 19680 & 40080 \\
\hline$\Delta t$ [ms] & 0.1 & 0.05 & 0.02 \\
\hline
\end{tabular}

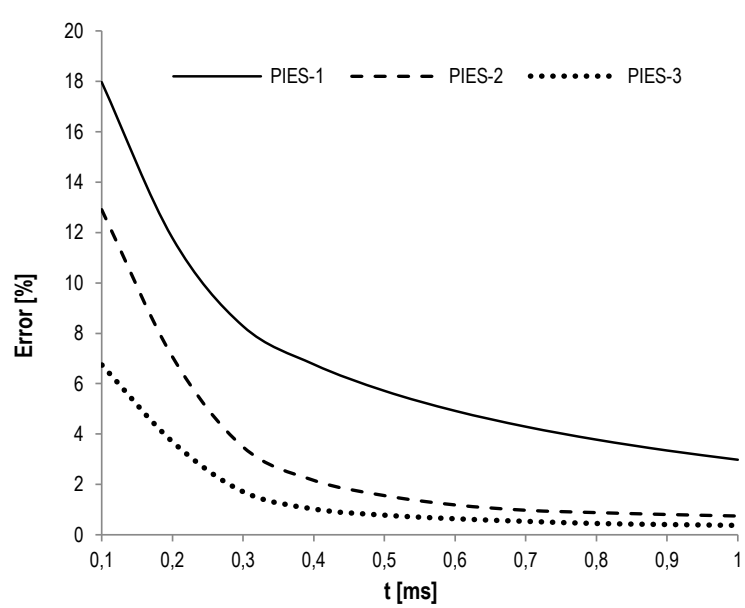

Fig. 3. Relative error [\%] history at point $p 1$, obtained by different PIES solutions, compared to the exact solution

Tab. 3. Input data for FEM

\begin{tabular}{|l|c|c|c|}
\hline & FEM-1 & FEM-2 & FEM-3 \\
\hline elements & 3249 & 6415 & 13293 \\
\hline nodes & 9976 & 19564 & 40336 \\
\hline$\Delta t[\mathrm{~ms}]$ & 0.1 & 0.05 & 0.02 \\
\hline
\end{tabular}

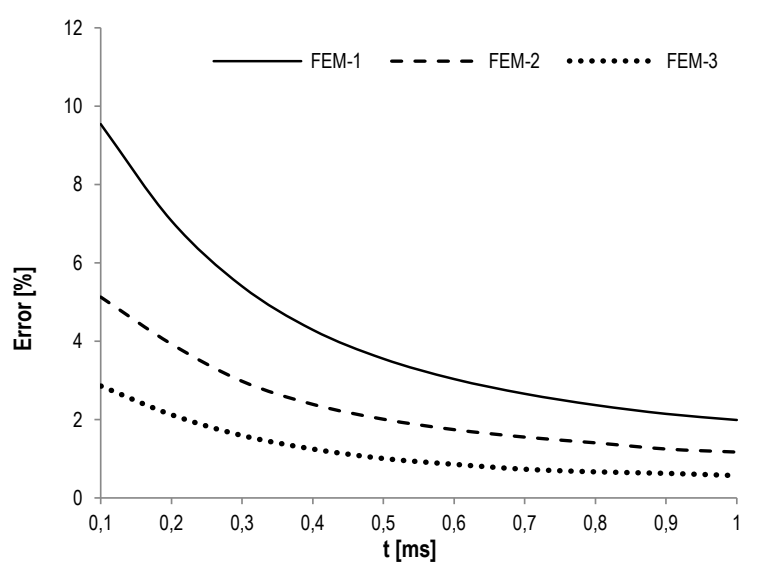

Fig. 4. Relative error [\%] history at point $p 1$ obtained by different FEM solutions, compared to the exact solution

FEM (Fig. 4) also gives more accurate results for smaller time step size and larger number of nodes (elements).

Tab. 4 presents temperature value at the point $p 1$ and time $t_{s}$. Temperature has been obtained for the exact solution, best PIES solution and best FEM solution.
Tab. 4. Temperature at point $p 1$ and time $t_{s}$

\begin{tabular}{|c|c|c|c|}
\hline $\mathrm{t}[\mathrm{ms}]$ & Exact & PIES-3 & FEM-3 \\
\hline 1 & 723.10 & 725.81 & 718.97 \\
\hline
\end{tabular}

Taking into account the data presented in Fig. 3-4 it can be noticed that FEM gives more accurate results than PIES, which is especially visible in the beginning of simulation. Tab. 4 shows that for $t=t_{s}=1[\mathrm{~ms}]$, best FEM and PIES solutions give results with similar accuracy.

In the next step, the best PIES solution and the best FEM solution have been compared. A longer time period has been considered, including the time after which the laser has been turned off. Fig. 5 presents temperature history at point $p 1$, obtained with both methods during the laser heating process and after the laser has been turned off.

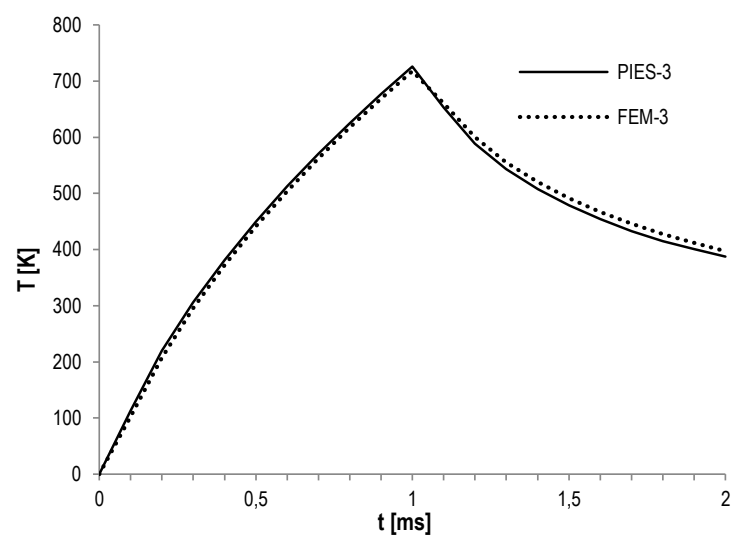

Fig. 5. Temperature history at point $p 1$ obtained with best PIES and FEM solutions for rectangular beam shape

As can be noticed, both methods give close results during the whole simulation time.

\subsection{Triangular pulse shape}

Next, a simulation for the triangular pulse has been carried out (7). The exact solution for this particular problem does not exist, so the problem has been first solved with FEM and then with PIES. Results obtained with PIES have been compared to the best FEM results. In FEM a different number of elements, nodes and different time step size have been considered, as presented in Tab. 3. Results are presented on Fig. 6. Result is the temperature history at point $p 1$.

As it can be seen on Fig. 6, improve of the accuracy of FEM solutions by reduction of the time step size and increase of the number of elements (nodes) leads to stabilization of results. FEM3 has been chosen as the best solution.

Sequentially, the example has been solved with the use of PIES method and results have been compared to the best FEM solution (FEM-3). Parameters of specific PIES solutions can be found in Tab. 2. Temperature history at point $p 1$ obtained with PIES can be found on Fig. 7 .

Fig. 7 shows that increase of accuracy of PIES method brings the obtained results closer to results obtained with best FEM solution. Closest to the FEM-3 results were the best PIES results (PIES-3). 


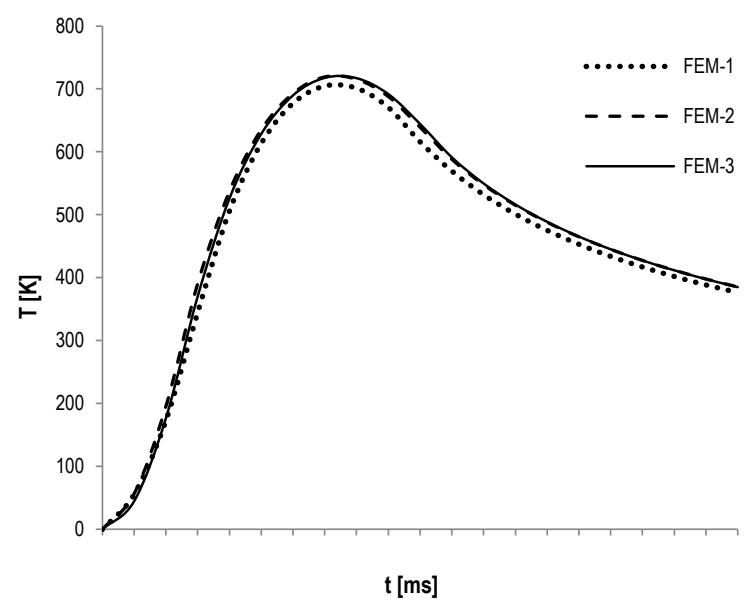

Fig. 6. Temperature history at point $p 1$ obtained with FEM for triangular pulse shape

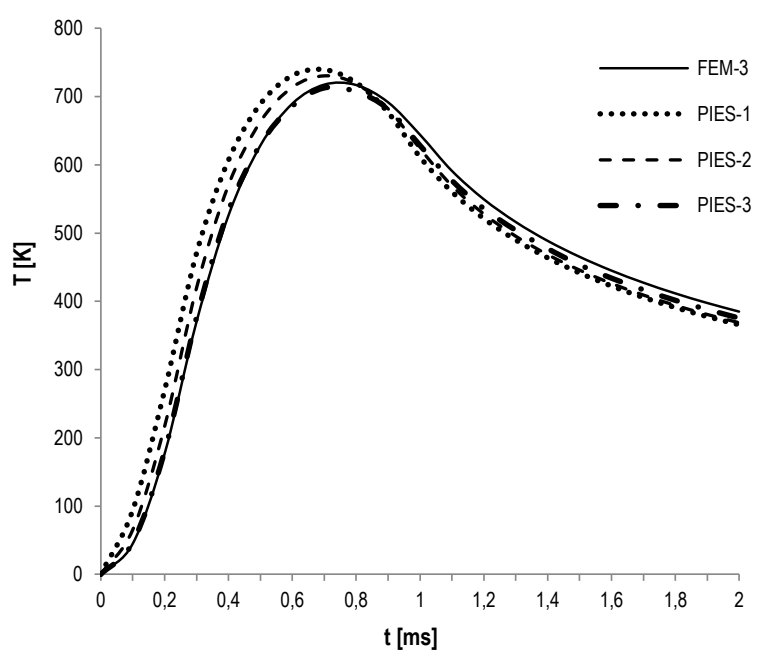

Fig. 7. Temperature history at point $p 1$ obtained with PIES and compared to best FEM solution, for triangular pulse shape

\subsection{Gaussian pulse shape}

In the next step, the Gaussian approximation function (8) has been used in the simulation. The same procedure has been used here, as in previous example. Due to the lack of exact solution, examples has been solved first with FEM and best FEM solution was used as a reference points for results obtained with PIES method.

Different variants of FEM (Tab. 3) have been considered. Results are presented on Fig. 8. Result is the temperature history at point $p 1$.

As it can be seen on Fig. 8, also in this case, increase of the accuracy of FEM solutions results in stabilization of obtained results.

Next, the example has been solved with PIES method. Obtained results are compared with FEM-3 results.

As can be noticed (Fig. 9), increase of accuracy in PIES makes the results obtained with this method closer to the best FEM results.

Fig. 10 presents the temperature history at point $p 1$, obtained with best PIES-3 solution, for three different laser pulse shape approximation functions (6-8).

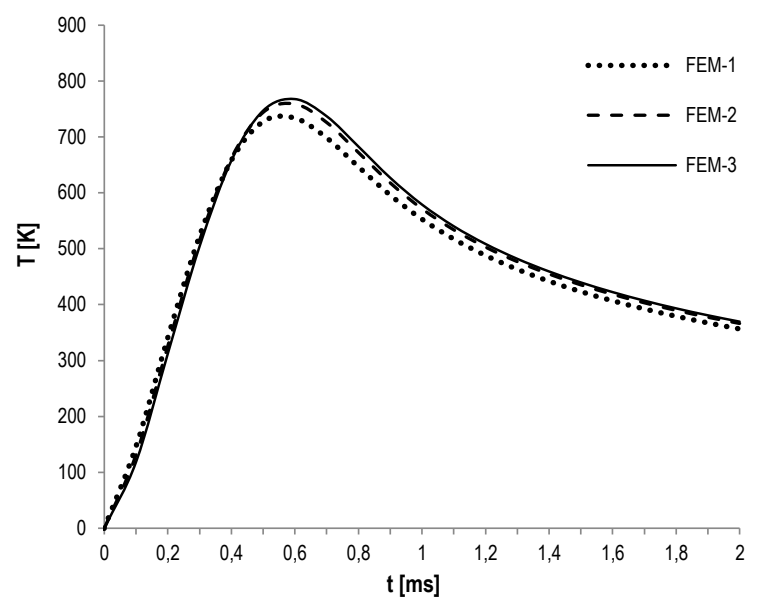

Fig. 8. Temperature history at point $p 1$ obtained with FEM for Gaussian pulse shape

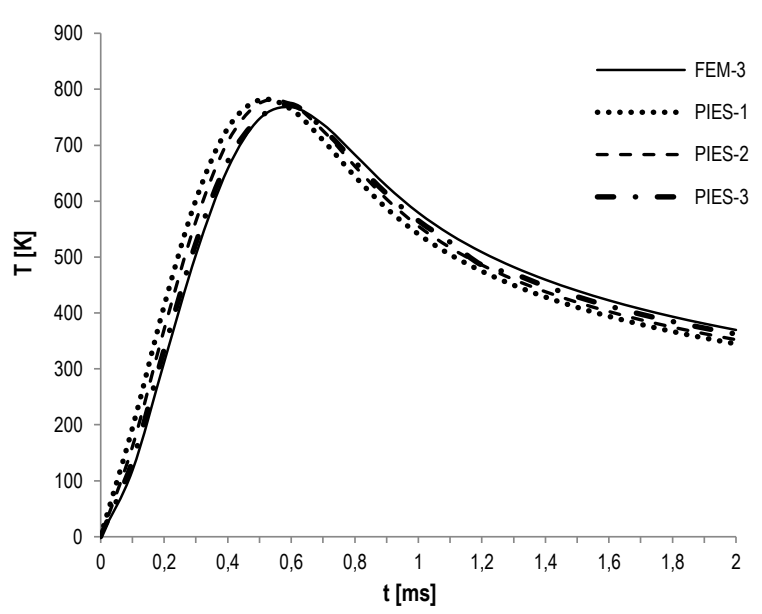

Fig. 9. Temperature history at point $p 1$ obtained with PIES and compared to best FEM solution, for Gaussian pulse shape

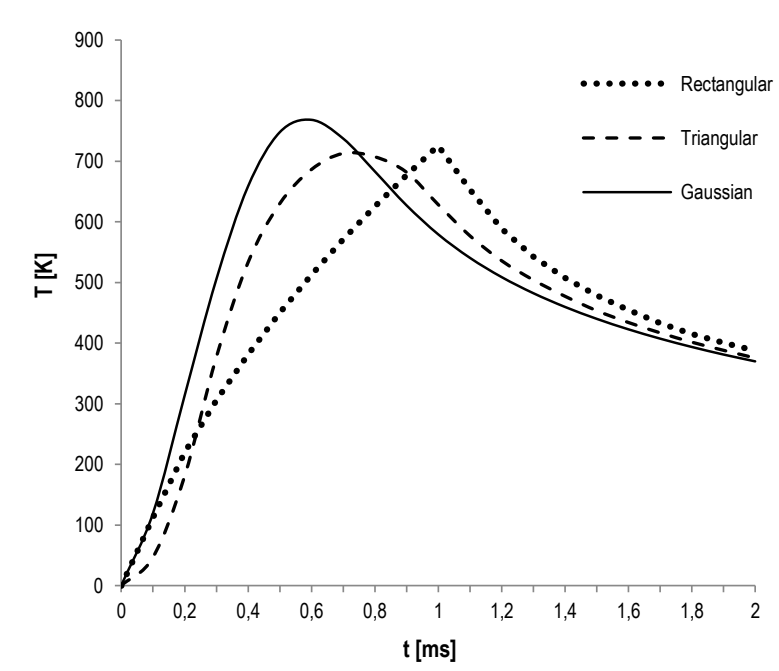

Fig. 10. Temperature history at point $p 1$ obtained with best PIES solution, for three different pulse shapes

As can be seen on Fig. 10, the temperature history obtained for different pulse shape approximation functions varies, which is mostly visible at the beginning of simulation. After some time the temperature stabilizes and all graphs converge to a single point. 


\section{CONCLUSIONS}

This paper presents the parametric intergral equations system for temperature equation, with the use of time stepping scheme. In the time stepping scheme each step is treated as a seperate problem, which allows in an easy way to solve issues with all boundary condition dependent on time. The exaples presented in this paper, laser heating of homogeneous materials belong to this category of issues. Three different functions approximating the real laser pulse shape have been considered. Comparison of the results with the exact solution and FEM found that PIES method gives accurate results, comparable with FEM. Therefore, PIES method can be an alternative for the commonly used FEM. In the future, further tests are planned to prove the effectiveness of PIES method in solving transient heat transfer problems.

\section{REFERENCES}

1. Al-Nimr M. A., Alkam M., Arpaci V. (2002), Heat transfer mechanisms during short-pulse laser heating of two-layer composite thin films, Heat and Mass Transfer, 38( 7-8), 609-614.

2. Brebbia C. A., Telles J. C., Wrobel, L. C. (1984), Boundary element techniques, theory and applications in engineering, Springer, New York.

3. Brugger K. (1972), Exact solution for the temperature rise in a laserheated slab, J. Appl. Phys., 43, 557-583.

4. Gladush G. G., Smurov I. (2011), Physics of Laser Materials Processing: theory and experiment, Springer series in materials science, 146.

5. Jewtuszenko A., Matysiak S. J., Różniakowska M. (2009), The temperature and thermal stresses caused by the laser impact on construction materials (in Polish), OWPB, Białystok.

6. Jirousek J., Qin Q. H. (1996), Application of hybrid-Trefftz element approach to transient heat conduction analysis, Computers \& Structures, 58, 195-201.
7. Johanssona B. T., Lesnicb D. (2008), A method of fundamental solutions for transient heat conduction, Engineering Analysis with Boundary Elements, 32, 697-703.

8. Lewis R. W., Morgan K., Thomas H. R., Seetharamu K. (1996), The Finite Element Method in Heat Transfer Analysis, Wiley.

9. Majchrzak E. (2001), Boundary element method in heat transfer (in Polish), Wydawnictwo Politechniki Częstochowskiej, Częstochowa.

10. Nowak A. J., Brebbia C. A. (1989), The multiple-reciprocity method. A new approach for transforming BEM domain integrals to the boundary, Engineering Analysis with Boundary Elements, 6, 164-167.

11. Partridge P. W., Brebbia C. A., Wrobel L. C. (1992), The dual reciprocity boundary element method, Computational Mechanics Publications, Southampton.

12. Tanaka M., Matsumoto T., Yang Q. F. (1994), Time-stepping boundary element method applied to 2-D transient heat conduction problems, Applied Mathematical Modelling, 18, 569-576.

13. Warren R. E., Spark M. (1979), Laser heating of a slab having temperature-dependent surface absorption, J. Appl. Phys., 50, 79527958.

14. Xiaokun Z., Rui D., Hua W. (2011), A VBCM-RBF based meshless method for large deflection of thin plates, International Conference on Multimedia Technology (ICMT), Hangzhou, 2380-2384.

15. Yanez A., Alvarez J. C., Lopez A. J., Nicolas G., Perez J. A., Ramil A., Saavedra E. (2002), Modelling of temperature evolution on metals during laser hardening process, J. Appl. Surf. Sci., 186, 611-616.

16. Zieniuk E. (2013), Computational method PIES for solving boundary value problems (in Polish), Polish Scientific Publishers PWN, Warsaw.

17. Zieniuk E., Sawicki D., Bołtuć A. (2014), Parametric integral equations systems in 2D transient heat conduction analysis, International Journal of Heat and Mass Transfer, 78, 571-587. 